\title{
The First Blood Collection Attempt for Doping Control in an Olympic Tournament: Data on the Procedure Applied in Athens 2004 Olympic Games
}

\author{
Christina Tsitsimpikou ${ }^{*, 1,2}$, Athanasios Z. Jamurtas ${ }^{1,2}$, Athanasios Tsiokanos ${ }^{1,2}$, Ioannis G. Fa- \\ touros $^{3}$, Konstantinos Tsarouhas ${ }^{2}$, Eva Karachaliou ${ }^{1}$, Patrick Schamasch ${ }^{5}$ and Dimitris Valasiadis ${ }^{1,4}$ \\ ${ }^{1}$ Athens 2004 Organising Committee for the Olympic Games; ${ }^{2}$ Department of Physical Education, University of Thes- \\ saly, Karies, 42100, Trikala, Greece; ${ }^{3}$ Department of Physical Education, University of Thrace, Komotini, 69100, Ko- \\ motini ${ }^{4}$ Olympic Athletic Centre of Athens (OAKA), Kifissias 37, Maroussi, Athens, Greece; ${ }^{5}$ International Olympic \\ Committee
}

\begin{abstract}
The procedure for blood sampling in doping control, implemented for the first time as part of the official forensic testing programme in the Athens 2004 Summer Olympic Games is described.

743 whole blood and serum samples from 37 sports were analyzed. In serum human growth hormone and hemoglobin based oxygen carriers were screened for. In whole blood samples heterologous blood transfusion was detected. Routine haematological parameters (hematocrit, haemoglobin) were followed in a very limited range only in Modern Pentathlon under the responsibility of the respective International Federation.

The majority of the blood samples collected concerned events in athletics. Endurance sports accounted for $81 \%$ of the whole blood sampling. Athletics, swimming and rowing represented 55\% of the serum samples analyzed. Extension of testing for oxygen transport enhancing agents in anaerobic sports should be seriously considered by future organizers and the International Olympic Committee.

The mean time for an athlete to report to the Doping Control Station for blood collection was 6.1 hours; $27 \%$ of the athletes tested (from 19 sports) failed to appear within the 12-hours time limit set.

Training of the phlebotomists was proven effective. Only 25 athletes of those tested (3\%) reported to have felt uncomfortable. The majority of complaints (47\%) concerned the prolonged waiting period and the long blood collection time (48\%).

Finally, an earlier agreement between all involved parties, attempts to decrease the time to appear at the site of blood collection and shortening of the duration of blood collection and sealing of the samples are recommended.
\end{abstract}

Keywords: Sport, WADA, anti-doping, prohibited substances, blood sampling.

\section{INTRODUCTION}

Doping Control, as part of the forensic sciences, has been an integral element of many international events over the last three decades and today is performed routinely in various official athletic events and the Olympic Games.

Urine had been the biological material of choice for Doping Control for many years, since its collection is noninvasive, relatively easy to perform and the window of detection for most classes of prohibited substances is dramatically extended even to weeks depending on the administration route in comparison to other biological material, such as blood $[1,2]$.

Nevertheless, in recent years, some international sports federations, such as the International Biathlon Union in 1994, have introduced blood testing procedures that can lead

*Address correspondence to this author at the Department of Physical Education and Sport Science, University of Thessaly, 15, Lakonias Str., 15122, Athens, Greece; Tel: +30-697-2004574; Fax: +30-210-6479303; E-mail: chtsitsi@yahoo.com to suspension from competition for athletes, whose haematological values exceed certain established limits, but not sanctioning. These health tests are not considered a part of the forensic doping control program and constitute a three-phase blood testing programme used to safeguard athletes' health and ensure fair competition $[3,4]$.

As science advanced, blood serum turned out to be a very promising biological material for the detection of human growth hormone (hGH) [5,6] and Haemoglobin-Based Oxygen Carriers (HBOCs) [7], while whole blood specimens could be used for the detection of heterologous blood transfusions (HBT) [8]. Blood collection was still not part of the Doping Control programme, until the Sydney Summer Olympic Games and Salt Lake Winter Olympic Games, where it was applied in a pilot scale, as International Olympic Committee (IOC) decided to use the haematological testing (hematocrit-Hct, reticulocyte hematocrit-RetHct, serum erythropoietin-EPO, percent macrocytes-\%Macro, soluble transferrin receptor-sTfr) to indirectly reveal athletes abusing recombinant erythropoietin (rEPO) [9] in combination with the direct detection of rEPO in urine [10]. 
Table 1. Summary of the Doping Control Program Applied in the Athens 2004 Summer Olympic Games

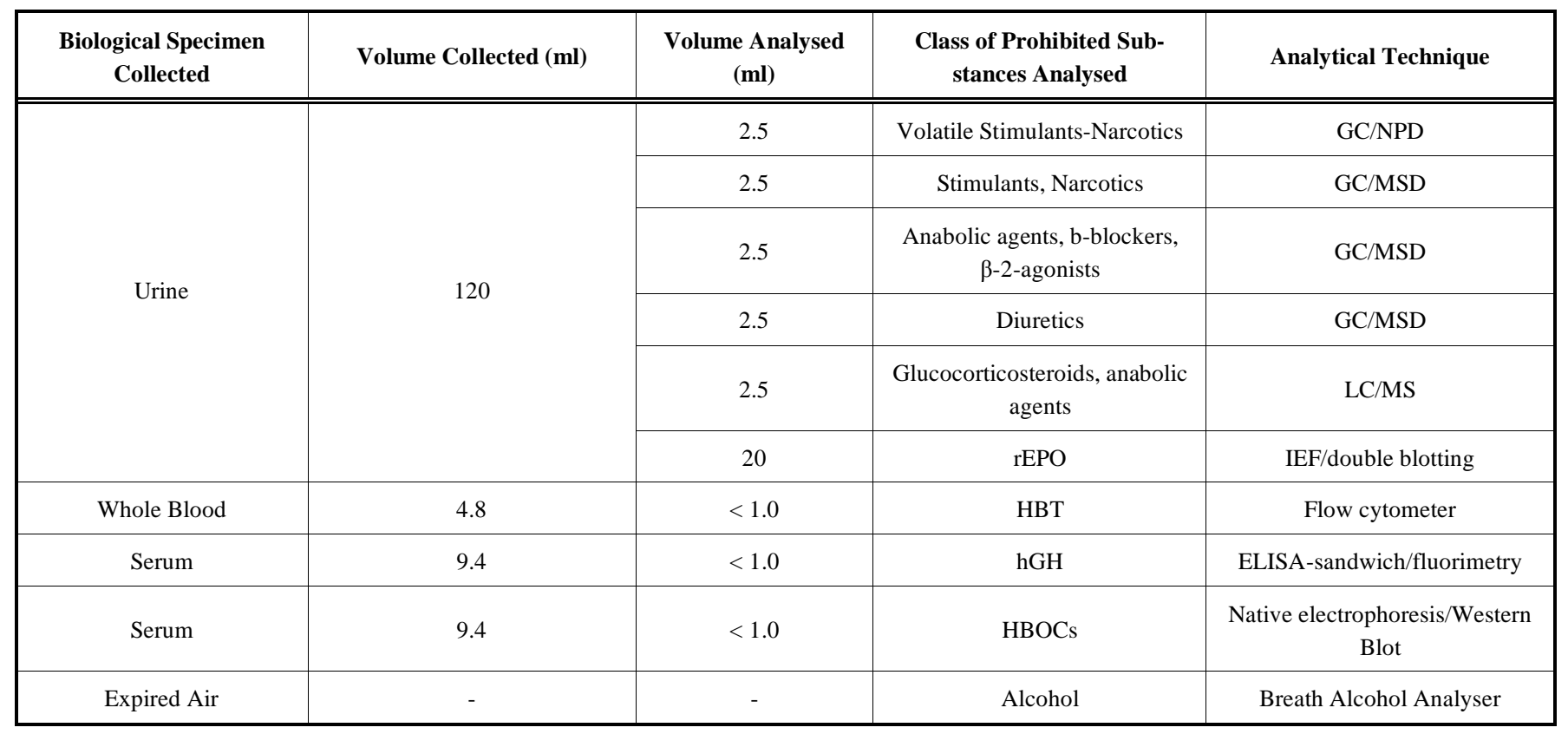

During the Athens 2004 Summer Olympic Games, blood samples were collected for the first time on an official basis as part of the forensic Doping Control programme implemented by IOC and analysis concerned the detection of prohibited substances and methods that could lead to Adverse Analytical Findings (AAFs) and possible sanctioning of the athlete [11-13]. The purpose of this paper is to describe the blood collection procedure adopted, the novelties introduced and to report on the athletes' attitude towards the procedure. Furthermore, statistical data regarding selection for blood testing during the last Olympic Games are presented. This report could contribute to the improvement of blood collection procedures for Doping Control in major international athletic events.

\section{MATERIALS AND METHODOLOGY}

\section{Agreements and Preparatory Considerations}

The sample collection procedure during the Athens Olympic Games was carried out according to the International Standard for Testing [12] and in general conformity to the Models of Best Practice for Urine Collection and Blood Collection of the World Anti-Doping Code of WADA [14]. The distribution of testing during the Athens 2004 Summer Olympic Games per sport was agreed in a separate agreement protocol with the respective International Federation (IF), IOC and Doping Control Services of Athens Organising Committee for the Olympic Games (ATHOC) and included testing in all competition rounds of at least the first four ranked athletes. The selection of athletes per National Olympic Committee (NOC) was based on the number of participating athletes per NOC, the placement of the athletes on the corresponding IF's ranking list, their availability for testing in the past, the results of previous testing, and the general information provided by WADA to the tripartite team consisting of IOC, WADA and ATHOC. This tripartite team was introduced for the first time in the history of Olympic Games.

\section{Participants}

10,862 athletes participated in the Athens 2004 Summer Olympic Games. The Doping Control Program comprised collection of urine, blood and expired air samples (Table 1). 3,527 athletes were tested for Doping Control. 743 whole blood and serum samples were analysed from 37 different Olympic sport disciplines. 679 of them were part of the Doping Control Program in combination with urine samples and the rest were part of the health test program.

\section{Blood Collection Process}

Following notification at the competition site (venue), athletes had to report to the Doping Control Station (DCS) for blood drawing. Blood collection took place within a maximum of 12 hours from completion of competition [8].

Phlebotomists hired for the Athens 2004 Summer Olympic Games were trained intensively in order to avoid unpleasant or traumatic incidents during blood collection. Special training focused on the venipuncture technique [15] and the use of the Vacutainer system (S-Monovette ${ }^{\circledR}$, Sarstedt, Germany), as well as the pathophysiology of the side-effects of phlebotomy. The aforementioned system was chosen due to the advantages it presented when taking multiple blood samples: the puncture and the first sample could be collected with the pre-assembled needle using the aspiration principle, depending on the condition of the athlete's vein. The gentle aspiration technique has clear advantages, especially in view of the difficult vein conditions often encountered in e.g. paediatrics, geriatrics or oncology. All subsequent tubes could then, if the vein's condition allowed, be filled using the vacuum technique. The needle remained in the vein, while tubes were changed, allowing leak-free multiple sampling.

There was not a preference as far as the dominant or nondominant arm to be used as no such requirement was set by the analytical methods to be applied [6-8]. Tourniquet was used and the time of holding wasn't standardized, since there 
aren't any references proving its effect on the analysis, as it could be the case during the measurement of Hct and hemoglobin $(\mathrm{Hgb})$ for health tests $[7,16]$.

Vacutainers of $4.7 \mathrm{ml}$ were used to collect blood for serum separation. The $4.7 \mathrm{ml}$ tubes contained gel beads in order to permanently separate serum from the cellular particles of the blood after centrifugation, as well as a coagulation activator. Whole blood samples were collected in Vacutainers of $2.4 \mathrm{ml}$ containing di-potassium ethylenediamine tetraacetic acid $\left(\mathrm{K}_{2}\right.$-EDTA) salts as an anticoagulant. The same syringes were used as tubes, and thus the pouring of the sample into another tube for sealing and transfer to the Laboratory was avoided. When a blood sample was to be tested only for serum hGH, two samples of $4.7 \mathrm{ml}$ were collected in two different tubes from the athlete $(9.4 \mathrm{ml}$ of blood in total). In all other cases, $14.2 \mathrm{ml}$ of blood (two times 2.4 $\mathrm{ml}$ samples of whole blood plus two times $4.7 \mathrm{ml}$ samples for serum separation) were collected in different tubes. An athlete was venipunctured only once, as the needles had a multiple adjustable insert. In the case where the phlebotomist failed, three consecutive times to locate an athlete's vein, which never occurred, the athlete had the right to refuse to continue the process.

The two whole blood or serum samples collected from the same athlete were sealed and designated as A and B. A BEREG kit (Berlinger Special AG) was used for uniquely identifying and sealing the blood samples (Picture 1). The BEREG kit contains one glass bottle with security cap Asample (orange bottle label) and one glass bottle with security cap B-sample (blue bottle label). Each bottle is presealed in transparent shrink-sleeve wrap with a tear-downtag for easy opening. Every kit has a unique number, which is laser-engraved on the bottles and embossed on the caps. As soon as the bottle is closed properly, the sample is sealed and secure.

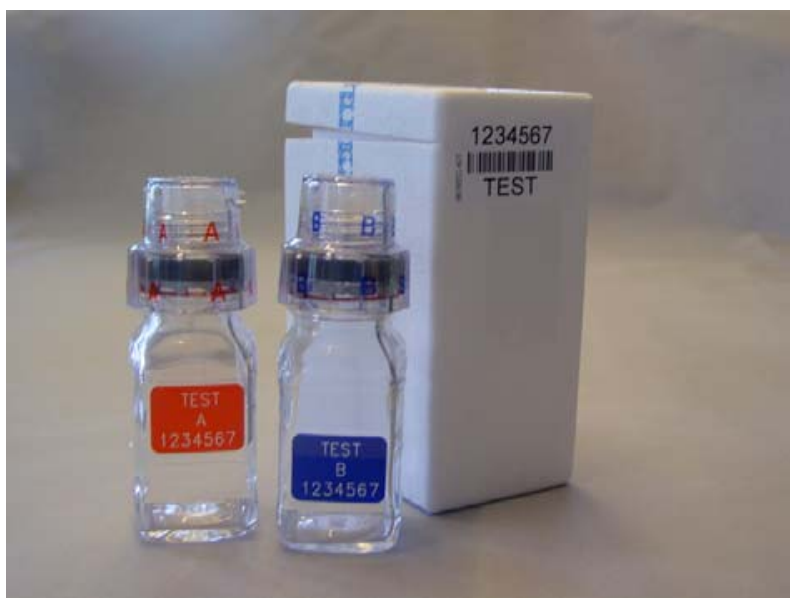

Picture (1). The BEREG kit used for uniquely identifying and sealing the blood samples.

Following blood collection, the whole blood samples tubes were placed directly into two bottles of the BEREG kit designated as A and B. Samples for serum separation were let to stand for 20 minutes at room temperature before centrifugation and then sealed along with the whole blood samples. The entire process took place in front of the athletes. The BEREG bottles with the blood samples in them were then placed in a refrigerator at $4^{\circ} \mathrm{C}$ for at least $30 \mathrm{~min}$ before packing in the styrofoam box of the kit, which during blood collection was not part of the kit and did not bear the sample code number.

Blood collection lasted maximum 32 min, when serum samples were involved.

The Blood Doping Control Official Record (BDCOR) was completed immediately after the sample collection process (Picture 2). It recorded the full name of the athlete, gender, age, date and time of sample collection, medication/ food supplements taken by the athlete the last three days prior sample collection, declaration of any blood transfusions received by the athlete over the last six months prior sample collection, specimen information and classes of prohibited substances analysed and the sample code of the BEREG kit. The athlete was entitled to record his observations on the process on this form. The athlete, his/her representative, the Doping Control Officer, the phlebotomist, the IOC and the IF representative (if present) signed the BDCOR. The BDCOR was produced in multiple coloured copies and each copy was delivered to all involved parties. The copy of the BDCOR accompanying the sample to the Laboratory ensured the athlete's anonymity during the analysis as it contained no information on the athlete and only the sample code of the BEREG kit. Upon completion of the Doping Control procedure at the DCS, the styrofoam boxes with the samples were delivered to the Doping Control Laboratory, sealed in plastic bags, within $20 \mathrm{~min}$.

\section{Measurements}

Following a decision by the IOC, ATHOC agreed to carry out testing for hGH and HBOCs in serum, as well as detection of HBT in whole blood specimens, in athletes of all sports. Testing for hGH concerned all athletes submitted to pre-competition testing (379), as well as gold-medal winners from all Olympic sports. Testing for HBOCs and HBT concerned the first four of the final rankings of all sports classified as aerobic (Table 2). Medallists from sports and disciplines classified as non-aerobic were also chosen by lot at the DCS in the presence of a representative from the corresponding IF before the beginning of the final phase of each sport/discipline.

The foregoing was communicated to the IFs and the NOCs on July 292004 through a circular letter signed by the IOC Medical Commission Director and the ATHOC Doping Control Programme Manager, which was distributed to the NOC pigeonholes at the Olympic Village and to the Presidents of the IFs.

Furthermore, on orders of the International Modern Pentathlon Federation (UIPM), 64 routine blood analyses for haematological parameters under the responsibility of UIPM were conducted as a health test programme following the same blood collection procedure.

\section{Results and Discussion}

This is the first time that information regarding the blood collection for Doping Control purposes in Olympic Games is presented. The blood collection procedure to be adopted was new for sports organisations. Hence, only two Federations (Shooting and Softball) agreed on the protocol between ATHOC, IOC and IF in 2003, whereas the UIPM agreed on 


\section{ATHOC \\ AӨHNA 2004}

Doping Control Official Record / Procès-verbal de Contrôle du Dopage

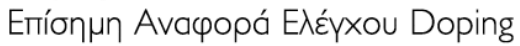

\section{Blood / Sang / Aíra}
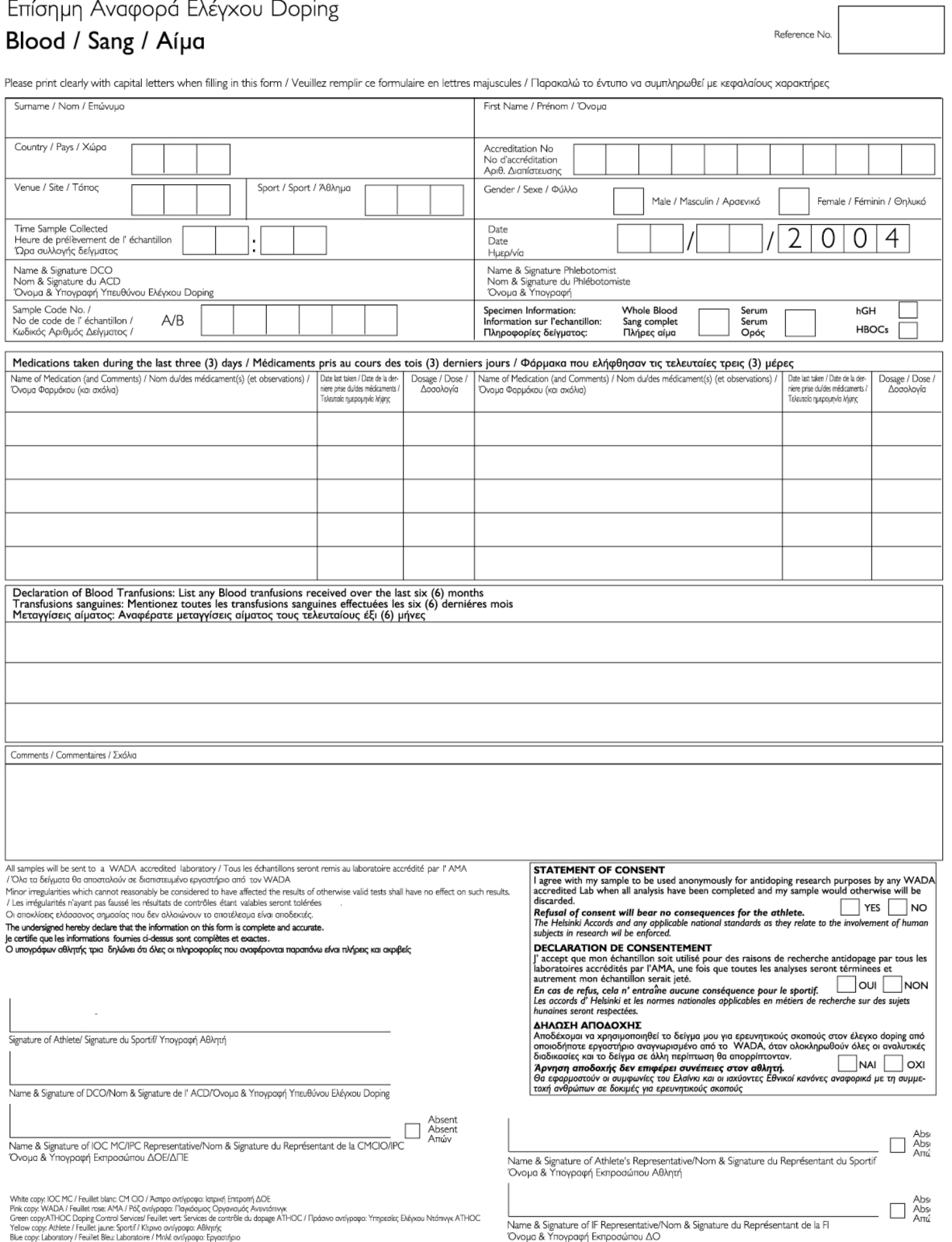

Picture (2). The Blood Doping Control Official Record used in Athens 2004 Summer Olympic Games.

August $6^{\text {th }} 2004$, a few days prior to the beginning of the Olympic Games. 24\% of the IFs signed the protocol agreement 5 months prior the start of the Games and $38 \%$ only 4 months before. A prompt agreement would have facilitated both the organisers and the scientists involved and at the same time would have helped the athlete get familiarised with the Doping Control procedure (s)he should attend.
Blood collection in major events involves structural and procedural organization. One major concern is the location of the blood collection site [11]. The inclusion of blood collection facilities in the DCSs at the venues changed three times during the preparation period for the Athens 2004 Summer Olympic Games. Finally, ATHOC, adapting to the IOC decision in May 2004 to officially introduce blood test- 
Table 2. Sports and Disciplines Regarded as Aerobic During the Athens 2004 Summer Olympic Games

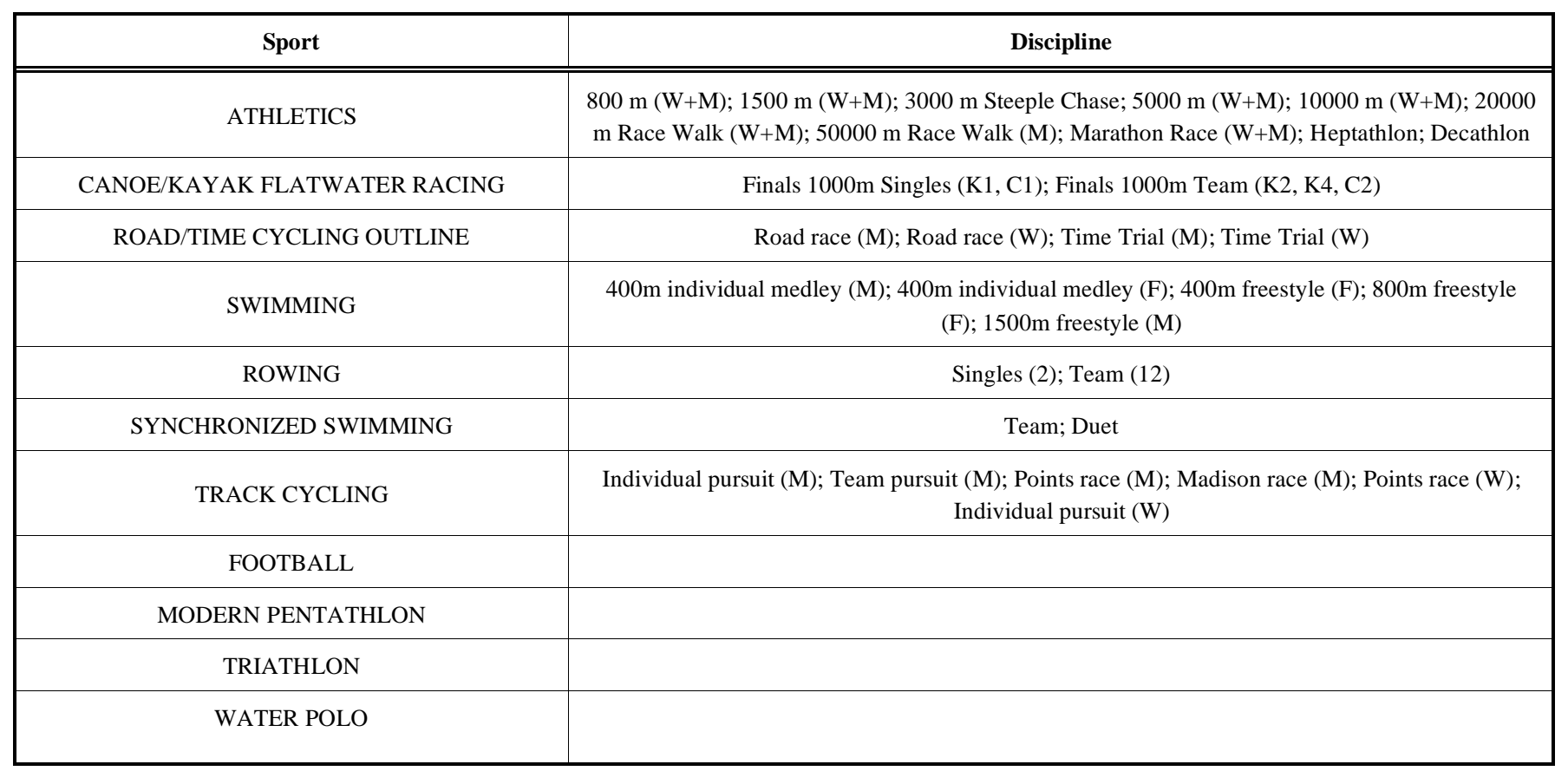

ing into the Doping Control programme of the Athens 2004 Summer Olympic Games, modified the construction only of the Polyclinic's DCS, in order to create suitable Doping Control blood processing areas. At the same time, and in order to fulfil requests made by the respective International Federations (FISA, ICF) and various NOCs during Olympic Games-time, the DCS of the Olympic Rowing and Canoeing Centre was temporarily modified for blood collection during the final phase of the sports of rowing (RO) and canoe-kayak flatwater (CF) (21-22 and 27-28 August, respectively). A similar course of action was followed on the $29^{\text {th }}$ of August 2004 at the Olympic Aquatics Centre and the Olympic Stadium to cover the needs of blood collection of the Men's water polo finals (8 samples) and Men's marathon (4 samples), respectively. In this case, the Athens 2004 Summer Olympic Games testing had to be completed within the $29^{\text {th }}$ of August, at which point the IOC's responsibility ended, making the transportation of the athletes to the Olympic Village inadvisable.

It is interesting enough that when the option was available to the athletes of RO and CF to conduct blood collection either at the Schinia competition venue or at the Olympic Village Polyclinic Doping Control Station (OLV), all CF blood testing was conducted at the OLV, whereas in RO, 50$71 \%$ of the testing took place at the OLV. This could mean that the athletes prefer to book appointments for blood collection at their Olympic residence instead of finishing all their Doping Control responsibilities at the venue, probably due to the fact that at the OLV several other medical facilities were available to the athlete at the same time. On the other hand, the competition schedule of RO and CF concluded early in the afternoon of each competition day, allowing enough time to the athletes to submit to blood and urine collection for doping control.

Nearly half (48.6\%) of the blood collections took place in the afternoon (13:00-18:00), while only $16.5 \%$ were con- ducted after 18:00 hours and 34.9\% in the morning (08:0013:00). The mean time for an athlete to report to the DCS for blood collection was $6.1 \mathrm{~h}$ (ranging from $5 \mathrm{~min}$ to $24 \mathrm{~h}$ ). Almost one third of the athletes $(35.2 \%)$ reported to the station within one hour after the notification and another $20 \%$ within two hours. However, $27.3 \%$ of the athletes (from 19 different sports) failed to appear within the 12-hours time limit set for valid reasons. It appears that three sports (athletics, road cycling and swimming) accounted for $62.4 \%$ of the athletes that failed to report within the desired time, whereas $85.4 \%$ is covered by seven sports. The main reasons include the heavy competition schedule, the fact that the athletes and the athletes' support personnel were not familiar with the procedure and the reluctancy of the athlete to submit to blood collection.

In Athens 2004 Summer Olympic Games, the percentage of testing on blood samples reached $23 \%$ of total testing (urine, blood and expired air). The time of blood collection procedure was approximately 35 minutes due to the centrifugation and the coagulation before the sealing of the sample in front of the athlete. Development of a new kit that could be compatible with the sealing specifications, while, at the same time, allowing the centrifugation in the sealed container will further reduce this time.

The percentage of athletes per sport in relation to the total number of participants subjected to blood testing is shown in Fig. (1). The results indicate that the majority of the samples come from athletes participating in aerobic sports, perhaps because the transfer of the oxygen is of greater importance for these sports. However, as anecdotal reports indicate, athletes participating in non-aerobic sports also tend to use several oxygen transport enhancing agents, it would be interesting to examine trends of use of illegal supplements that enhance the oxygen carrying ability in athletes of anaerobic sports. Therefore, future organizers and IOC should seriously consider this for future events. 


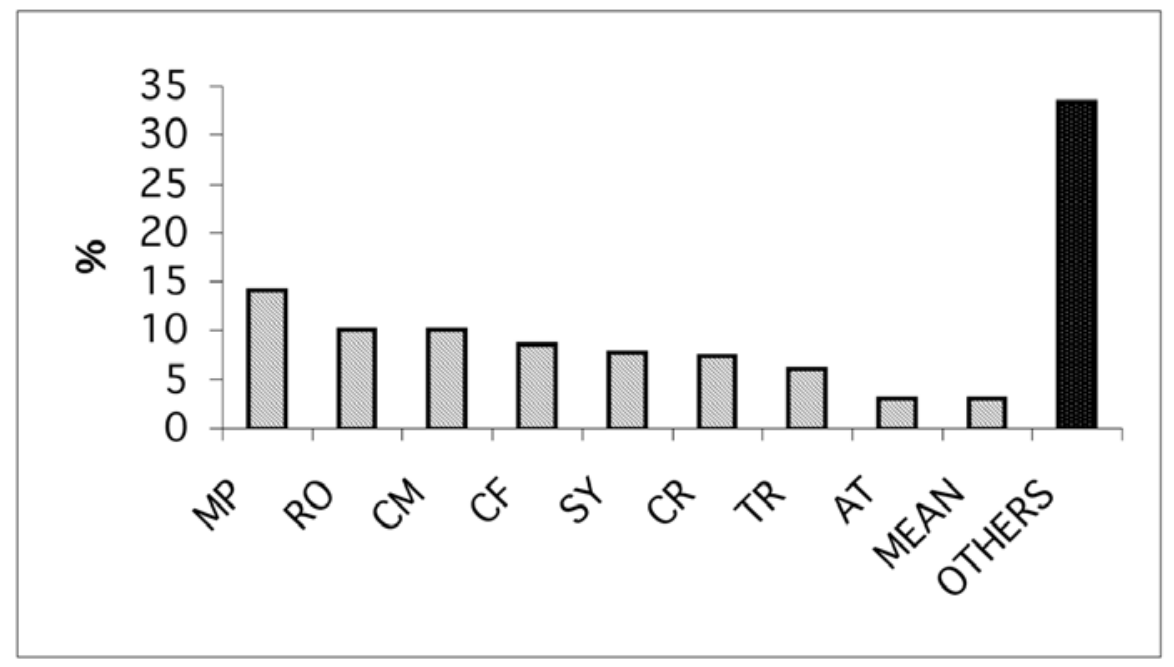

Fig. (1). Percentage of athletes per sport in relation to the total number of participants subjected to blood testing MP: Modern Pentathlon; RO: Rowing; CM: Mountainbike; CF: Canoe Kayak; SY: Synchronised Swimming; CR: Road Cycling; TR: Triathlon; AT: Athletics.

On the BDCOR there was a specific field where the athlete was entitled to record his/her observations on the process. In general, the outcome of a newly procedure tested in such a major event with numerous samples to be collected from different venues was satisfactory given that only 25 athletes (3\%) felt uncomfortable with the procedure and only two athletes made really dissatisfactory remarks regarding the blood sampling procedure. Furthermore, there was only one case (a female weightlifter) that the appointment for blood collection had to be rescheduled due to problems during phlebotomy. The main reason for the athletes' discontent (48\%) was the prolonged waiting period in the waiting room and the long blood collection time. Other reasons included problems with the tourniquet while sampling and other minor procedural non-conformities (20\%), unsuccessful venipuncture or limited sample collection due to vein problems $(16 \%)$, preference of the athletes to conduct blood collection in parallel with urine collection at the competition venues (8\%). All the above should be taken into account in future blood Doping Control programs. The most dissatisfied athletes as shown by their comments on the DCOR for blood collection came from the Netherlands and Australia (20\% and $12 \%$, respectively), while athletics, swimming and rowing produced almost $50 \%$ of the negative comments. Negative comments were equally divided between pre- and postcompetition tests. It is interesting that none of the wellknown side effects of phlebotomy, such as nerve injury, vasovagal reaction, hematoma, allergy, infections and anxiety associated with blood and injections causing fainting symptoms $[16,17]$ were recorded or associated with blood collection either at the DCS or at the Polyclinic of the Olympic Village. Therefore, the procedure applied was proven to be on the whole safe, with the frequency and / or severity of side effects being low.

It is worth noting that neither the IOC Medical Commissioner nor the IF representatives attended the blood collection procedure at the venues, with one exception in rowing, fencing and diving and 11 exceptions in swimming, despite the fact that the whole procedure was entirely novel and implemented for the first time in the Olympic Games. Only in one case in diving, involving an unsuccessful venipuncture on a female Canadian diver during the pre-competition period, the respective IF representative was present to supervise the procedure. Both IOC and the individual federations should embrace similar efforts in order to improve these weaknesses.

Finally, for the first time as part of the BDCOR, the athlete was asked to consent to the use of his/her sample for research purposes. Seventy five percent of the athletes consented. Only athletes from badmington and basketball unanimously denied use of their samples for research, probably following informal guidelines from their respective IFs. On the other hand, athletes competing in $30 \%$ of the Olympic sports, like tennis and table tennis, gymnastics, volleyball etc, unanimously consented to the use of their sample for research.

\section{CONCLUSION}

The results showed that even though there were no significant problems with the collection, an earlier agreement between all the involved parties would ease the process. A prompt agreement would have facilitated both the organisers and the scientists involved and at the same time would have helped the athlete get familiarised with the Doping Control procedure (s)he should attend. Location of the collection site proved to be equally important, as athletes preferred the OLV in the Polyclinic probably because access to other medical facilities was available at the same time. Future organizers should take under consideration the vast variability in the reporting time for blood collection and the great number of athletes that did not report within the time limit set and try to find means to decrease both the time of appearance at the site of blood collection and the number of athletes that fail to report within the prescribed time. Another concern should be the duration of blood collection and sealing of the samples. Development of a new kit, which could be compatible with the sealing specifications, while, allowing the centrifugation in the sealed container, will further reduce this time. In addition, wider extension of testing for several oxygen transport enhancing agents in athletes of anaerobic 
sports is also suggested to future organizers and the IOC. To conclude with, the procedure applied was proven to be on the whole efficient, acceptable, safe and with the frequency and / or severity of side effects being low.

\section{REFERENCES}

[1] Maurer, H.H. Systematic toxicological analysis procedures for acidic drugs and/or metabolites relevant to clinical and forensic toxicology and/or doping control. J. Chromatogr. B Biomed. Sci. Appl., 1999, 733, 3-25.

[2] Kintz, P.; Villain, M.; Cirimele, V. Hair analysis for drug detection. Ther. Drug Monit., 2006, 28, 442-446.

[3] Manfredini, F.; Carrabre, J.E.; Litmanen, H.; Zhukovskaja, L.; Malagoni, A.M.; Dal Follo, D.; Haberstroh, J. Blood tests and fair competition: the biathlon experience. Int. J. Sports Med., 2003, 24, 352-358.

[4] Robinson, N.; Schattenberg, L.; Zorzoli, M.; Mangin, P.; Saugy, M. Haematological analysis conducted at the departure of the Tour de France 2001. Int. J. Sports Med., 2005, 26, 200-207.

[5] Saugy, M.; Robinson, N.; Saudan, C.; Baume, N.; Avois, L.; Mangin, P. Human growth hormone doping in sport. Br. J. Sports Med., 2006, 40, i35-i39.

[6] Bidlingmaier, M.; Strasburger, C.J. Technology insight: detecting growth hormone abuse in Athletes. Nat. Clin. Pract. Endocrinol. Metab., 2007, 3, 769-777.

[7] Lasne, F.; Crepin, N.; Ashenden, M.; Audran, M.; de Ceaurriz, J. Detection of hemoglobin-based oxygen carriers in human serum for doping analysis: screening by electrophoresis. Clin. Chem., 2004, $50,410-415$
[8] Nelson, M.; Popp, H.; Sharpe, K.; Ashenden, M. Proof of homologous blood transfusion through quantification of blood group antigens. Haematologica, 2003, 88, 1284-1295.

[9] Sharpe, K.; Hopkins, W.; Emslie, K.R.; Howe, C.; Trout, G.J. Kazlauskas, R.; Ashenden, M.J.; Go, C.J.; Parisotto, R.; Hahn, A.G. Development of reference ranges in elite Athletes for markers of altered erythropoiesis. Haematologica, 2002, 87, 1248-1257.

[10] Lasne, F.; Martin, L.; Crepin, N.; de Ceaurriz, J. Detection of isoelectric profiles of erythropoietin in urine: differentiation of natural and administered recombinant hormones. Anal. Biochem., 2002, 311, 119-126.

[11] World Anti-Doping Agency: Independent Observers Report: Olympic Summer Games 2004. http:www.wada-ama.org [October 2004].

[12] World Anti-Doping Agency: International Standard for Testing 4.0. http:www.wada-ama.org. [March 2004].

[13] World Anti-Doping Agency: International Standard List of Prohibited Substances and Methods. http:www.wada-ama.org [March 17, 2004].

[14] World Anti-Doping Agency: The World Anti-Doping Code. http:www.wada-ama.org.

[15] Little, M. A.; Hussein, T.; Lambert, M.; Stuart, J. Percutaneous venepuncture practice in a large urban teaching hospital. Clin. Med., 2007, 7, 243-249.

[16] Deacon, B.; Abramowitz, J. Fear of needles and vasovagal reactions among phlebotomy patients. J. Anxiety Disord., 2006, 20, 946-960.

[17] Ohnishi, H. Side effects of phlebotomy: pathophysiology, diagnosis, treatment and prophylaxis. Rinsho Byori., 2005, 53, 904-910.

(c) Tsitsimpikou et al.; Licensee Bentham Open.

This is an open access article distributed under the terms of the Creative Commons Attribution License (http://creativecommons.org/licenses/by/2.5/), which permits unrestrictive use, distribution, and reproduction in any medium, provided the original work is properly cited. 